\title{
Applied Behavior Analysis And The Treatment of Childhood Psychopathology And Developmental Disabilities
}

\author{
JOEL E. RINGDAHL and TERRY S. FALCOMATA
}

\section{INTRODUCTION}

This chapter provides a description and examples of the use of applied behavior analysis (ABA) in the treatment of childhood psychopathology and developmental disabilities. This task is a daunting one given that many of the single topics that are discussed in the following pages can, and have, served as topics for entire chapters and texts. This limitation means we are not able to delve into each topic in a comprehensive manner. However, we do provide an overview of the important topics related to ABA and its use in the treatment of childhood psychopathology and developmental disabilities. In addition, we provide a discussion of literature-based examples for these ABA-based treatments, brief examples of generalization of treatment effects, and discussion of effectiveness.

It is important to note that ABA is not a single treatment. It is more accurate to say that $\mathrm{ABA}$ represents an approach to treatment as opposed to a specific type of treatment. This approach includes a number of treatment strategies that can be used to address the behavioral symptoms associated with childhood psychopathology and the behavioral challenges

JOEL E. RINGDAHL and TERRY S. FALCOMATA - The University of lowa. 
associated with developmental disabilities. As an approach to treatment, ABA relies on arranging response-consequence relationships (including positive and negative reinforcement and punishment), schedule of reinforcement effects, and antecedent manipulations (including stimulus-control procedures and altering establishing operations) to reduce problem behavior and increase appropriate behavior.

In the paragraphs that follow, we provide an overview of ABA, including how it is defined, a description of ABA-based treatment strategies, including both consequence and antecedent-based interventions, and a brief summary of recent effectiveness research related to ABA-based treatments and behavior problems common to individuals with childhood psychopathology and/or developmental disabilities.

\section{APPLIED BEHAVIOR ANALYSIS DEFINED}

Baer, Wolf, and Risley (1968) outlined seven dimensions of applied behavior analysis. It is upon these dimensions that the clinical applications are based. According to Baer et al., ABA is applied, behavioral, and analytic. In addition, ABA should be technological, conceptually systematic, effective, and generalizable. The term applied indicates that the target behavior is of social significance. It is the emphasis on social significance that sets ABA apart from laboratory analysis. Examples of applied are wideranging and can include any behavior that society deems important. The term behavioral indicates the focus of ABA should be on actions exhibited by the individual as opposed to what the individual says about those actions. Pragmatically, the implication is that what should be measured are observable actions exhibited by an individual. The term analytic indicates that a "believable demonstration of events ... responsible for the occurrence or non-occurrence of that behavior" (p. 93-94). Thus, ABA approaches to treatment are often implemented and demonstrated within a single-subject research design (e.g., reversal, multielement, or multiple baseline designs).

Along with establishing what constituted applied, behavior, and analytic, Baer et al. delineated four other dimensions for ABA. Applied behavior analysis should be technological, meaning that the "techniques making up a particular behavior application are completely identified" (p. 95). Thus, it is incumbent upon the behavior analyst implementing an ABA-based treatment to provide a complete description of its components. Baer et al. also asserted that ABA be conceptually relevant explanation. Additionally, Baer et al. stressed practical significance, as opposed to theoretical significance, as a hallmark of ABA. In essence, if a behavioral technique does not produce effects that are of practical value, that application has failed. Finally, behavior change produced by ABA should be durable over time (i.e., effects should be generalizable).

Since the publication of Bear et al.'s dimensions of applied behavior analysis, other behavior analysts have described additional characteristics of ABA. Heward (2005) described ABA as accountable, public, doable, empowering, and optimistic. Cooper, Heron, and Heward (2007) described these characteristics in the following manner. 
Accountable: "The commitment of applied behavior analysis to effectiveness, their focus on accessible environmental variables that reliably influence behavior, and their reliance on direct and frequent measurement to detect changes in behavior yield an inescapable and socially valuable form or accountability" (p. 18).

Public: "... ABA is visible and public, explicit and straightforward" (p.18) Applied behavior analysis is transparent and there are no hidden or unexplained treatments.

Doable: the interventions found to be effective in ABA studies are able to be implemented by "teachers, caregivers, coaches, supervisors, and sometimes even the individuals themselves" (p.19). Cooper et al. suggest that the procedures are not "prohibitively complicated or arduous" (p. 19).

Empowering: "ABA gives practitioners real tools that work" (p. 19) thus improving their confidence.

Optimistic: the result of practitioners having effective strategies and the ability to detect improvements, along with literature-based examples of success gives cause for optimism regarding the future success of behavior change programs.

Collectively, Cooper et al. (2007) summarized these dimensions and characteristics of applied behavior analysis as "... the science in which tactics derived from the principles of behavior are applied systematically to improve socially significant behavior and experimentation is used to identify the variables responsible for behavior change" (p. 20). This reliance on systematic evaluation of the variables responsible for behavior change results in an approach to the assessment and treatment of behavior problems that is functional, as opposed to structural. Thus, the selected treatment, or treatment package, is based on the relationship demonstrated between the presenting behavior of interest (i.e., out of seat behavior) and the environment. This approach can be contrasted to an approach that prescribes or selects treatment based on the diagnosis (e.g., $\mathrm{ADHD}$ ) that is of concern. Using this functional approach, it is conceivable that the same treatment(s) could be used to address different presenting concerns and different treatment(s) might be used to address similar presenting concerns.

\section{DESCRIPTION OF ABA TREATMENT APPROACHES}

In this section, an overview of many of the frequently used ABA-based treatments is provided. The section has been subdivided into consequence-based treatments (reinforcement and punishment based), antecedent-based treatments (noncontingent reinforcement and prompting strategies), and treatments in combination (i.e., including two or more consequence or antecedent components or at least one antecedent and one consequence-based component).

Also included in this section are examples in application of each of the treatment strategies described. At least two examples from the literature are 
provided for each treatment. When possible, an example is provided for both childhood psychopathology (or disorders not associated with developmental disabilities) and developmental disabilities. Given that the ABA approach has been most widely used to treat the psychopathologies of children in two broad categories (early childhood disorders such as conduct disorder, disruptive behavior disorder, and attention-deficit/hyperactivity disorder and anxiety and phobias), childhood psychopathology examples will likely fit into one of these two categories. The developmental disabilities examples focus on the treatment of behavioral challenges presented by individuals with developmental disabilities and autism. These challenges include, but are not limited to (1) problems of behavioral excess such as stereotypic movement disorder, self-injurious behavior, aggression, destruction, tantrums, and so on, and (2) problems of behavioral deficit such as delays in language development, difficulty with skill acquisition, and problems with academic performance.

\section{Consequence-Based Procedures: Punishment}

Punishment is a response-dependent (i.e., contingent) operation resulting in the decreased likelihood of a particular response. Two types of punishment have been described in the behavior analysis literature: positive and negative. Positive punishment involves the response-dependent delivery of a stimulus that results in a subsequent decrease in responding. Alternatively, negative punishment involves the response-dependent removal of a stimulus that results in a subsequent decrease in responding. The effect on behavior is the same; the difference stems from the action given to the stimulus (i.e., presented or removed).

\section{Positive Punishment}

As indicated, positive punishment involves the contingent presentation of an aversive stimulus following the target response. In application, this approach to treatment has included any number of aversive stimuli including, but not limited to, aversive outcomes such as electric shock (Linscheid, Iwata, Ricketts, Williams, \& Griffin, 1990), water mist (Singh, Watson, \& Winton, 1986), facial screen (Rush, Crockett, \& Hagopian, 2001), aversive activities such as exercise (Kahng, Abt, Wilder, 2001), and overcorrection (Foxx \& Azrin, 1973).

Linscheid et al. (1990) described the treatment of self-injurious behavior (SIB) exhibited by five individuals, including three individuals under the age of 18, with developmental disabilities. It is important to note that each of the five cases had a long-standing history of SIB that had proven unmanageable and was severe in nature (i.e., caused significant tissue damage or put the individual at risk of tissue damage or death). As well, the authors address issues related to generalization, maintenance, and potential for abuse for this particular treatment. Treatment included the contingent application of electric shock following occurrences of severe SIB. Immediate and pronounced effects were observed for each of the five participants. Anecdotal follow-up data suggested that no habituation had occurred for four of the five participants months after treatment was initiated. 
Kahng et al. (2001) described the implementation of a positive punishment procedure to reduce the SIB exhibited by a 16-year-old girl. One topography of SIB was reduced by the implementation of a noncontingent reinforcement procedure. However, other topographies of SIB continued to be exhibited when this treatment procedure was in place. As a second treatment component, an aversive activity (i.e., exercise; touching toes) was made contingent on each occurrence of all topographies of SIB. This procedure was added to the ongoing noncontingent reinforcement program as well as a restraint fading program. Immediate reductions in SIB were observed when this punisher was in place.

\section{Negative Punishment}

Negative punishment involves the contingent removal of a reinforcer following occurrences of the target response. Applied examples of the procedure include response cost and timeout from reinforcement. Response cost is the loss of a specific amount of a reinforcer following each occurrence of the target response, resulting in a decreased probability of the response (Cooper et al., 2007).

Conyers et al. (2004) used a response cost procedure to reduce the disruptive behavior exhibited by 25 children in a classroom setting. Specifically, the authors compared a reinforcement-based procedure (differential reinforcement of other behavior; DRO) with response cost. During $\mathrm{RC}$, each child's name was displayed on a board and 15 stars (tokens) were placed next to each name. Disruptive behavior resulted in the loss of a token. The remaining tokens could be traded for preferred items at the conclusion of each session. Results of the study suggested that, although both RC and DRO behavior were effective in reducing disruptive behavior, the classroomwide RC procedure was more effective.

Long, Miltenberger, and Rapp (1999) incorporated response cost into a treatment package to reduce the thumb sucking and hair pulling exhibited by a typically developing six-year-old girl. Reinforcement-based procedures were ineffective in reducing the behavior to sufficiently low levels. Thus, a response cost contingency was added to the reinforcement package. Specifically, the participant was able to earn an M\&M at specific time intervals for engaging in behavior other than thumb sucking or hair pulling. When the RC component was added, the participant was told she would lose one M\&M for engaging in either thumb sucking or hair pulling. Immediate reductions of both these target responses were observed. According to the authors, the participant only lost access to one M\&M during the first session of treatment with the RC contingency in place. Treatment gains were maintained for 23 weeks. Corresponding decreases in problem behavior were reported by the participant's parents in the home setting.

Time out from reinforcement (TO) includes the "withdrawal of the opportunity to earn positive reinforcers or the loss of access to positive reinforcers for a specified time, contingent on the occurrence of a behavior" (p. 357). Again, the effect on behavior is decreased probability of future occurrence (Cooper et al., 2007). 
Kodak, Grow, and Northup (2004) used time out from reinforcement as a component of treatment to reduce the elopement exhibited by a young child diagnosed with ADHD. A functional analysis of the child's elopement behavior indicated it was maintained by adult attention. During treatment, this consequence (adult attention) was provided on a scheduled basis (every 15s). However, if the child engaged in the target response (elopement), she was removed from the activity for $30 \mathrm{~s}$ and adult attention was withheld. This combination of components resulted in a decrease in elopement to near-zero levels.

Falcomata, Roane, Hovanetz, Kettering, and Keeney (2004) implemented a time out from reinforcement procedure to reduce the inappropriate vocalizations exhibited by an 18-year-old individual with developmental disabilities. The researchers were able to identify a highly preferred activity (i.e., a positive reinforcer, listening to the radio), and access to this activity was interrupted for a specified time following occurrences of the target behavior. The timeout contingency resulted in almost immediate reductions in problem behavior. Any number of studies could have been included here to illustrate the effects of timeout from reinforcement in application. The Falcomata et al. study was included because it illustrates the close relationship between $\mathrm{RC}$ and time out from reinforcement. Many researchers in applied behavior analysis do not draw a distinction between the two treatments (in fact, the title of the Falcomata et al. article is "Response cost in the treatment of ..."). The take-home point is that both $\mathrm{RC}$ and TO involve contingent removal of positive reinforcers.

There are several concerns that go along with the use of punishment. Vollmer (2002) discussed four potential concerns regarding the use of punishment that are often raised. First, punishment procedures can sometimes produce negative emotional side-effects. Second, the effects of punishment are often short-lived. Third, punishment procedures have the potential to be abused. This risk of abuse, to some, outweighs the benefits of some procedures. Finally, the treatment does not teach the individual an appropriate behavior that can be used to recruit reinforcers from their environment. Additional concerns regarding the use of punishment include the development of escape and avoidance behavior, behavioral contrast (i.e., an increase in the behavior targeted for punishment in the absence of the punisher), and undesirable modeling (Cooper et al., 2007).

It is important to note that neither Vollmer (2002) nor Cooper et al. (2007) advocate against the use of punishment procedures. Instead, they provide discussions of some of the considerations that need to be taken into consideration before developing and implementing a punishment-based procedure. However, for the above stated reasons, and, often because of administrative and legal reasons, reinforcement-based strategies are typically implemented as a first step in the treatment of behavior problems.

\section{Consequence-Based Intervention Strategies: Reinforcement}

Reinforcement involves the response-dependent delivery (positive reinforcement) or removal (negative reinforcement) of a stimulus, resulting in an increased future likelihood of the target response. Reinforcement-based 
procedures often serve as the cornerstone for both simple and complex behavior-change programs. In application, reinforcement-based procedures include such strategies as token economies, contingency contracting, and differential reinforcement. In each approach, a consequence is identified using some sort of selection process including preference assessments, reinforcer assestsments, or functional analyses of target behavior. The stimulus or stimuli identified via these procedures are then scheduled for delivery contingent on the behavior targeted for increase. Delivery can take place after each occurrence of the behavior, after a specified number of occurrences, following the first response after a specified time interval (i.e., the stimuli are delivered on ratio or interval schedules), or in a deferred manner once some behavioral criteria are met (i.e., the stimuli are delivered as part of a token economy). In addition, a single response can be targeted for increase, or a sequence of responses can be targeted.

\section{Positive Reinforcement}

Positive reinforcement procedures involve the contingent delivery of a known preferred item or reinforcer contingent on a behavior targeted for increase. When delivered on a ratio or interval schedule, the individual must meet a particular response requirement (e.g., two responses or one response after $10 \mathrm{~s}$ has elapsed) to gain access to the positive reinforcer. This strategy is most often used when the clinical goal is the establishment of an appropriate behavior, such as communication or task completion, or a repertoire of appropriate behavior such as social skills or toileting.

Graff, Gibson, and Galiatsatos (2006) used a positive-reinforcement procedure to increase the vocational and academic work completed by four adolescents with developmental disabilities. In this study, high and low preferred stimuli were identified via a series of preference assessments. High preferred and low preferred stimuli were then made contingent on completion of various vocational tasks. The results of the study demonstrated that the contingent presentation of both high and low preferred stimuli increased the rate of vocational responses. However, contingent presentation of the high preferred stimuli was correlated with higher, sustained response rates for each participant.

Luiselli (1991) described the use of a positive reinforcement procedure to increase the independent feeding behavior of a boy with Lowe's syndrome. Specifically, praise and access to sensory-based reinforcers (i.e., light and music stimulation) was provided contingent on independently completing components of the self-feeding response. As each component was mastered, the reinforcer was provided for the next response in the task analysis. Results indicated that the participant exhibited acquisition of each of the steps of the task analysis, eventually exhibiting independent self-feeding.

\section{Negative Reinforcement}

Negative reinforcement procedures involve the contingent removal (escape) of an aversive event, or allow the individual to postpone an aversive event (avoidance). When delivered on a ratio or interval schedule, 
the individual must meet a particular response requirement (e.g., two responses or one response after $10 \mathrm{~s}$ has elapsed) before the negative reinforcer is removed or postponed. This strategy is most often used when an individual exhibits problem behavior maintained by escape or avoidance of instructional, self-care, or other aversive situations.

Kelley, Piazza, Fisher, and Oberdorff (2003) increased the number of cup sips exhibited by a child with a feeding disorder through the application of a negative reinforcement procedure. Prior to treatment, nonpreferred foods were identified via a stimulus preference assessment. During treatment, spoonfuls of the nonpreferred food were presented along with prompts to take sips from a cup. Sips from the cup allowed the child to escape the bite of nonpreferred food that was otherwise presented if inappropriate behavior or sip refusal was exhibited. Increases in sips were observed as a function of this negative-reinforcement procedure.

Rolider and Van Houten (1985) applied negative reinforcement to the treatment of encopresis associated with constipation exhibited by a 12year-old with no other stated diagnoses. During the negative reinforcement-based treatment, the participant was required to sit on the toilet for 20 min or until a bowel movement occurred. If the child had a bowel movement, she was not required to sit on the toilet again that day. If no bowel movement occurred, she was required to sit on the toilet for $40 \mathrm{~min}$ or until a bowel movement occurred at the next scheduled toilet sitting. Defecation resulted in no more sitting that day. If no bowel movement occurred, she was required to sit for $90 \mathrm{~min}$ or until a bowel movement occurred at the next scheduled toilet sitting. Any bowel movement outside of scheduled toilet sittings also resulted in the child being able to avoid the remaining toilet sittings for the remainder of that day. The treatment resulted in increased levels of successful bowel movements on the toilet.

\section{Token Economy}

A token economy involves the delivery of a conditioned reinforcer (e.g., a token, point, or other stimulus) that can later be exchanged for another reinforcer. According to Cooper et al. (2007), token economies consist of three components including a list of target behavior or responses, tokens or points that will be earned for exhibiting the target response(s), and a menu of items or activities for which the points or tokens can be exchanged. When implementing a token economy, considerations need to be made regarding the conditioning of the tokens, the menu of backup or primary reinforcers, and the schedule with which the backup reinforcers are accessed. Breakdowns in any of these areas can reduce the effectiveness of the procedure. For example, if the tokens are not explicitly tied to the backup reinforcer(s), they will not affect the individual's behavior. Similarly, if the menu or backup reinforcers include nonpreferred stimuli, are arbitrarily selected (e.g., without the use of a stimulus preference assessment), or the stimuli are only available on a very lean schedule, the effect of the program could be limited. Token economies are often used in large group settings such as classrooms, residential treatment centers, and group-living environments. 
Field, Nash, Handwerk, and Friman (2004) implemented a token economy to decrease the inappropriate behavior (or, conversely, to increase the appropriate behavior) displayed by three children living in a residential treatment center. Each child had been diagnosed with various psychiatric disorders including conduct problems, ADHD, and PTSD. The existing, centerwide token economy was in place for each participant; however, the children's behavior continued to be unacceptable. The experimenters increased the schedule of token exchanges from a single exchange each day to two exchanges per day. This change in scheduled exchanges resulted in both a decrease in problem behavior and an increase in the percentage of exchange opportunities during which the participants earned the backup reinforcer.

Mangus, Henderson, and French (1986) described the use of a token economy system to improve the on-task physical activity time exhibited by children with autism in an educational setting. In their study, a peer tutor was trained to deliver a token on a predetermined schedule (the schedule differed for each of the five participants, based on the participants' performance during the last three days of baseline data collection) contingent on engagement in a physical education activity (i.e., walking on a balance beam). Tokens could be exchanged for edible reinforcers from a reinforcement menu once five tokens had been earned. Results of the study indicated that the on-task, physical activity of four of the five participants varied as a function of the token economy intervention. Specifically, when the token economy was in place, higher levels of on-task physical activity were observed. When the token economy was not in place, lower levels of on-task physical activity were observed.

\section{Consequence-Based Intervention Strategies: Extinction}

Extinction is a procedure that consists of the discontinuation of reinforcement for a behavior with a previous history of reinforcement for the purpose of reducing that behavior. In contrast to the variations of differential reinforcement (described later in this chapter), extinction-only procedures do not include reinforcement for alternative responses or decreases in the rate of the target response. Typically, the reinforcer that is withheld during any extinction procedure is one that has been identified as maintaining a target behavior (i.e., is a functional reinforcer).

Iwata, Pace, Kalsher, Cowdery, and Cataldo (1990) treated the escapemaintained SIB exhibited by six children with developmental disabilities. Treatment consisted of extinction and guiding the child through tasks contingent on occurrences of problem behavior (a response-blocking component was added for one participant), thus interrupting the aberrant response-reinforcement relationship identified during a functional analysis of the consequences maintaining problem behavior. Reductions in SIB were observed for each of the six participants. Compliance increased for five of the six participants (compliance data were not presented for the remaining participant), although compliance was not explicitly targeted for change (i.e., no consequences had been programmed for this response).

Magee and Ellis (2000) described the sequential application of extinction to the problem behavior exhibited by two children with attention-deficit/ 
hyperactivity disorder. One child's problem behavior (out of seat) was maintained by escape from task. This behavior decreased following the implementation of extinction. However, an increase in other behavior problems (yelling, inappropriate gestures, and destruction) was observed. Using a multiple baseline design, extinction was sequentially applied to each topography. A decrease in each topography was observed following the application of the extinction procedure. The second child's problem behavior was maintained by social positive reinforcement (attention). When the extinction procedure was first applied to object mouthing, that behavior decreased. However, increases were noted for two other responses, destruction and aggression. When extinction was implemented for each response, responding again decreased to near-zero levels.

Although these examples suggest that extinction can be an effective approach to treatment, its use has some limitations that preclude it from being used as the sole treatment component. First, implementing extinction can result in temporary increases in problem behavior at the outset of treatment (i.e., extinction burst), an outcome that can be especially problematic when treatment targets behavior that has the potential to cause injury. Second, extinction can lead to variations in response topography, including aggressive behavior.

To further evaluate these two drawbacks, Lerman, Iwata, and Wallace (1999) reviewed 41 data records for individuals whose treatments included an extinction component and for whom aggression was neither a target response nor programmed for reinforcement at any point during assessment. Their review identified extinction-induced response bursts for 39\% of the 41 reviewed cases. Similarly, Lerman et al. noted extinction-induced aggression in $22 \%$ of the data records included in their sample. A third drawback with extinction-only procedures is that they do not teach the individual alternative methods to obtain the reinforcer. Each of these three limitations can be addressed by including a differential reinforcement component to treatment. Differential reinforcement programs include contingent reinforcement of an alternative response, or the absence of the target response, is targeted for reinforcement, thus increasing the likelihood of an appropriate alternative behavior. This additional component can improve the effectiveness and limit the drawbacks associated with extinction-only procedures.

Again, looking at the data provided by Lerman et al., when the extinction-based procedure included a differential reinforcement, noncontingent reinforcement, of some antecedent manipulation as a component of treatment, extinction bursts were evident in only $15 \%$ of cases. Similarly, extinction-induced aggression was also only evident in $15 \%$ of cases when extinction was accompanied by other treatment components.

\section{Consequence-Based Intervention Strategies: Differential Reinforcement}

Differential reinforcement is a consequence-based procedure that consists of the reinforcement of one response class (i.e., a set of responses maintained by the same reinforcer or reinforcers) and withholding 
reinforcement for another response class (Cooper et al., 2007). Behavior analysts have developed several variations of differential reinforcementbased treatments. These treatment strategies are typically implemented to reduce a target problem behavior, whereas some include a component designed to increase a target appropriate behavior (e.g., compliance). When used for the purpose of reducing a target behavior, differential reinforcement involves two components: reinforcement of behavior(s) other than the target behavior or the reinforcement of decreasing rates of the target behavior, and withholding of reinforcement following the occurrence of the targeted problem behavior (Cooper, et al.).

Although behavior analysts often use differential reinforcement procedures for the purpose of reducing problem behaviors, it should be noted that differential reinforcement is also often used for the purpose of shaping new appropriate behaviors. As with all reinforcement-based procedures, differential reinforcement procedures can include positive or negative reinforcement.

\section{Differential Reinforcement of Alternative Behavior (Including Functional Communication Training)}

Differential reinforcement of alternative behavior (DRA) is a procedure that consists of the reinforcement of a specified behavior that is different from the behavior that has been targeted for reduction (but not necessarily incompatible with that target response). In a typical application, all occurrences of the behavior targeted for reduction are placed on extinction, and reinforcement is available for each appropriate response. One example of DRA treatment is functional communication training (FCT). This treatment consists of identifying the functional reinforcer responsible for the maintenance of problem behavior, and then delivering that reinforcer contingent on an appropriate communicative response (Carr \& Durand, 1985). When this procedure is implemented in such a manner that the reinforcer responsible for problem behavior is withheld (i.e., extinction is in place) contingent on that response or set of responses, it fits within the parameters of a DRA treatment.

It should be noted that researchers have compared the effectiveness of FCT with and without this extinction component. In one notable study, Hagopian, Fisher, Sullivan, Acquisto, and LeBlanc (1998) found that FCT without extinction was minimally effective for 11 participants. Some participants displayed a reduction in problem behavior, but none achieved a 90\% reduction. Three of the 11 participants exhibited an increase in problem behavior of atleast 50\% when FCT was conducted without extinction. By contrast, FCT with extinction was effective in achieving a 90\% reduction in $44 \%$ of applications ( 11 of 25 ). No increases in problem behavior were reported when FCT was implemented with an extinction component.

There have been several articles published regarding the utility of FCT in the behavior analytic literature. Derby et al. (1997) described the longterm effects of FCT as treatment for the problem behavior exhibited by four young children with developmental disabilities. Each of the children displayed reductions in target problem behavior and exhibited increases 
in or acquisition of appropriate communication following FCT implementation. This study demonstrated the robust effectiveness of FCT because the children's problem behavior was maintained by different functions (both positive and negative reinforcement), including one child whose problem behavior was maintained by multiple functions, and the treatment effects were observed across a longer than two-year time period.

Other DRA procedures focus on increasing appropriate behavior such as compliance with instructions. Reed, Ringdahl, Wacker, Barretto, and Andelman (2005) implemented differential reinforcement of alternative behavior to increase the compliance and decrease the problem behavior exhibited by two children with developmental disabilities. Each child's problem behavior was maintained by escape from tasks. During treatment, compliance with the tasks resulted in a $30 \mathrm{~s}$ break from instruction (i.e., negative reinforcement). Problem behavior resulted in immediate guidance through the task (i.e., extinction). For each child, compliance increased and problem behavior was reduced relative to baseline when the DRA treatment was in place.

\section{Differential Reinforcement of Incompatible Behaviors}

Differential reinforcement of incompatible behaviors (DRI) is a procedure that is very similar to DRA except that the designated alternative behavior targeted for reinforcement is incompatible with the behavior that has been targeted for reduction.

Friman and Altman (1990) implemented a DRI schedule to address the disruptive behavior exhibited by a 4-year-old boy with developmental disabilities. The target response for the child was out-of-seat behavior. During the treatment procedure, parents delivered reinforcers (praise and edibles) contingent on the child exhibiting an incompatible behavior (i.e., staying in his seat) for specified intervals (initially, 10s). If the child left his seat, he was reseated and the reinforcer was not delivered at the end of the interval. The treatment resulted in a decrease in out-of-seat behavior and a corresponding decrease in other inappropriate behavior (e.g., mouthing and throwing objects), whereas appropriate behavior (toy play) increased slightly.

Buzas, Ayllon, and Collins (1981) described the use of a DRI procedure to reduce the SIB (biting lip and tongue, picking at lips and mouth, biting inside of cheek, gouging tongue frenulum, and falling out of wheelchair) exhibited by a young boy with Lesch-Nyhan Syndrome. The participant's SIB was so severe that he spent the majority of his day in mechanical restraints. On the occasions when restraint was removed (for hygiene activities, dressing, etc.), almost immediate attempts at SIB were observed (e.g., tearing his lip with fingernail). In this case study, an array of responses incompatible with SIB (drawing, throwing plastic darts, playing games, doing puzzles, eating candy, playing catch, reading while holding a book, adding and subtracting numbers on a die, typing, wheeling his wheelchair, and learning sign language) resulted in access to attention from various therapists and caregivers.

When this DRI procedure was in place, the participant was able to interact out of restraint for up to three-and-a-half hours without 
attempting to engage in SIB. When the procedure was not in place and restraints were removed, attempts at SIB were observed within 5 to 15 minutes. Although this study is descriptive and lacks systematic experimental control, it is included here because of the clinically significant outcomes achieved. The behavioral problems associated with Lesch-Nyhan syndrome are notoriously resistant to treatment, both pharmacologic and behavioral in nature. One potential reason for this difficulty in treatment is that the reinforcers relevant to the behavior are unidentifiable or change too often to allow for systematic evaluation. The described study demonstrates the potential utility of arranging a differential reinforcement-based treatment when a reinforcing consequence can be identified and manipulated.

\section{Differential Reinforcement of Low Rates of Behavior}

Differential reinforcement of low rates of behavior (DRL) is a procedure that consists of the reinforcement of a behavior targeted for reduction but on a schedule of reinforcement that is leaner than what was in place prior to the implementation of the DRL procedure (i.e., the schedule of reinforcement in place in the natural environment). With the DRL procedure, the behavior targeted for reduction is reinforced only following a specified length of time in the absence of the behavior. In addition, as the reductions of the target behavior are observed over time, the length of the interval can be systematically increased in order to bring about lower and lower rates of the target behavior (often referred to as differential reinforcement of diminishing rates; DRD).

Wright and Vollmer (2002) reported the use of a DRL procedure to reduce the rapid eating exhibited by a teenage girl with developmental and physical disabilities. The procedure consisted of reinforcing bites (i.e., allowing access to the bite) only if bite attempts occurred on a predetermined interval. If the participant attempted to take a bite of food before the predetermined interval elapsed, that bite was blocked. If the participant attempted to take a bite of food after the predetermined interval elapsed, the bite was allowed (i.e., reinforced). The authors noted that the DRL procedure was more effective if the time interval was adjustable and based on the mean interresponse time (IRT) from the preceding five sessions than if it was fixed (i.e., 15s for every session). The DRL procedure resulted in longer time between bite attempts relative to baseline which translated to a decrease in the participant's bite rate.

Deitz and Repp (1973) reported a series of three experiments in which DRL schedules were used to reduce the disruptive behavior exhibited by a student diagnosed with a developmental disability, a classroom of students diagnosed with developmental disabilities, and a group of high school students enrolled in regular education. In each experiment, there was a decrease in disruptive behavior exhibited by either the target individual or the class as an aggregate when the DRL schedule was implemented. The treatment effects were maintained when the DRL schedule was withdrawn during Experiment I (single student). However, treatment effects were lost when the DRL schedules were withdrawn during Experiments II and III. 


\section{Differential Reinforcement of Other Behavior}

Although DRA, DRI, and DRL-based treatments target increasing a specific appropriate response along with reducing the target problem behavior, differential reinforcement of other behavior (DRO) consists of the delivery of reinforcers contingent on the absence of identified problem behavior for a specified time period. Whereas reinforcement involves the presentation of a stimulus contingent on a target behavior, DRO entails the contingent application of a reinforcing consequence (either positive or negative) for the nonoccurrence of a target behavior. Other terms that are sometimes used for DRO include differential reinforcement of the omission of behavior and differential reinforcement of zero rates of behavior.

Ringdahl et al. (2002) described the use of a DRO-based treatment to reduce the stereotypic hand movements exhibited by an adolescent boy with developmental disabilities. The stereotypic hand movements exhibited by the participant were serious in nature because they could trigger photosensitive grand mal seizures. The researchers were unable to identify any social reinforcers maintaining the problem behavior and noted that the behavior only occurred when adult supervision was not provided. A reinforcer assessment indicated that video games could potentially function as a reinforcer for appropriate behavior. Following assessment, access to video game time was allowed contingent on time intervals during which the participant did not engage in the target response. Initially, the DRO interval was set at $10 \mathrm{~s}$ (based on the average time between hand-flapping episodes during baseline) and gradually increased to $600 \mathrm{~s}$ ( $10 \mathrm{~min}$ ) by the end of treatment. Decreases in repetitive hand movements were observed when the DRO procedure was in place.

Watson and Sterling (1998) used a DRO procedure to reduce vocal tics exhibited by a 4-year-old girl. A functional analysis of this behavior indicated the vocal tics were maintained by social consequences in the form of adult attention. During treatment, adult attention was withheld or removed when vocal tics occurred. Alternatively, adult attention was provided following brief intervals $(15 \mathrm{~s})$ with no vocal tics. The interval was increased by $10 \mathrm{~s}$ following three consecutive deliveries of the reinforcer until the DRO interval reached the terminal length of $300 \mathrm{~s}(5 \mathrm{~min})$. A decrease in the rate of the vocal tic was observed when the DRO was implemented. This decrease was still apparent at one-, three-, and sixmonth follow-up visits.

\section{Thinning Differential Reinforcement Schedules}

One limitation of DR approaches to treatment, particularly DRA/FCT and DRI programs, is that the target individual can access reinforcers at any time contingent on appropriate behavior. If delivery of the reinforcer requires the presence of a caregiver, such programs can be labor intensive. As well, the individual may spend all of his time accessing the reinforcer, which can compete with academic or vocational goals. Thus, one goal of treatment is to reduce the availability of the reinforcer by increasing the response requirement or implementing a delay to reinforcement. 
Lalli et al. (1999) provided access to differential positive reinforcement contingent on compliance in the treatment of the escape-maintained problem behavior displayed by five individuals 21 years old or younger. The programmed positive reinforcement schedule resulted in increases in compliance and decreases in problem behavior exhibited by each participant. The response requirement to obtain the positive reinforcer was then increased for three of the five participants. At the outset of treatment, compliance resulted in positive reinforcement on a fixed-ratio (FR) 1 schedule. That schedule was increased to at least FR 10 for each of these three participants. This change in schedule did not result in degradation of treatment effects.

Hagopian, Contrucci Kuhn, Long, and Ruch (2005) implemented FCT for three boys diagnosed with PDD spectrum disorders admitted to an inpatient hospital setting for the assessment and treatment of severe behavior problems including aggression and disruption. Functional communication training resulted in decreases in problem behavior for each child. The authors thinned the schedule by implementing a delay between occurrences of appropriate requests and delivery of the reinforcer (attention or preferred tangible items). The delays were progressively increased if the participant exhibited less than 0.2 responses per minute (RPM) of problem behavior for two consecutive sessions at a given delay. If two sessions of greater than 0.2 RPM of problem behavior was observed, the delay was reduced to the previously longest successful delay. This progression continued until a terminal goal was met for each participant. For each of the three participants, delays of at least $4 \mathrm{~min}$ were achieved. One interesting finding from this study was that allowing the participants access to competing reinforcers during the delay interval allowed for quicker attainment of the terminal delay length and fewer occurrences of problem behavior.

\section{Antecedent Approaches to Treatment}

The majority of ABA treatments focus on manipulating consequences to change behavior, however, there are some treatments that focus on manipulating antecedents relevant to the target behavior. For the purposes of this chapter, four antecedent-based interventions are highlighted. These interventions include: procedures that manipulate establishing operations, stimulus control procedures, prompt procedures, and procedures that provide choice-making opportunities.

\section{Establishing Operations}

The relationship between environment and behavior is often described as a 3-term contingency. The three components of this contingency are what happens prior to the response (the antecedent, or A), the behavior the individual exhibits (B), and what happens immediately following the behavior (the consequence, or $\mathrm{C}$ ). Often, this 3-term contingency is denoted as A-B-C. A complete understanding of the antecedent requires that behavior analysts take into account variables that alter the effectiveness of a stimulus as a reinforcer. The term that has historically been used to describe this relationship between the environment and reinforcer 
effectiveness or value is "establishing operation" (EO; Michael, 1982). More recently, the term "establishing operation" has been replaced with the term "motivating operation" (MO) when the effect is an increase in the value of the reinforcer and the term "abolishing operation" (AO) when the effect is a decrease in the value of the reinforcer (Laraway, Snycerski, Michael, \& Poling, 2003). These operations (motivating or abolishing) affect behavior by either increasing (via an $\mathrm{MO}$ ) or decreasing (via an $\mathrm{AO}$ ) responding.

The most salient example of MOs and AOs are deprivation and satiation. Deprivation consists of withholding the stimulus that functions as a reinforcer from the individual. Deprivation has at least two effects. First, it results in an increase in the value of the stimulus as a reinforcer. Second, it results in an increase in responding that occurs as a function of that reinforcer. Conversely, satiation consists of presenting a sufficient amount of the stimulus that functions as a reinforcer and has two opposite effects: a decrease in the value of the reinforcer and a related decrease in responding that occurs as a function of that reinforcer.

In application, EOs can be manipulated in a number of ways. When attempting to decrease a target response, the identified reinforcer for that response can be provided on a noncontingent basis during treatment (i.e., reinforecers are delivered on a relatively dense fixed-time schedule; Ringdahl, Vollmer, Borrero, \& Connell, 2001). Alternatively, the functional reinforcer could be provided to the individual prior to exposing her to the context(s) in which the target response has historically been likely to occur (e.g., Vollmer \& Iwata, 1991; Berg et al., 2000). When attempting to increase a target response, the reinforcer can be withheld prior to training (e.g., Vollmer \& Iwata, 1991).

Lalli, Casey, and Kates (1997) used a fixed-time (FT) reinforcement schedule to reduce aberrant behavior exhibited by two children with mental retardation and one child with a developmental disability. The FT schedule specified when reinforcers were to be delivered. Delivery occurred independent of the child's behavior. The specific FT schedules used during treatment reflected the mean latency to problem behavior during baseline for each child. Decreased rates of problem behavior were observed with all three children when the FT schedule was implemented. One possible explanation for the decrease in aberrant behavior was that the FT schedule of reinforcement resulted in satiation indicating AO effects.

Taylor et al. (2005) manipulated the EO associated with preferred snacks to increase peer-directed mands (i.e., requests) exhibited by three children with autism. MOs were altered through the restriction of the preferred snacks and access to the snacks was made contingent on mands that were peer-directed. When the MO was in place, rates of peer-directed mands were observed at high rates. Conversely, when the MO was not in place, mands decreased to near-zero rates for each of the children. The results demonstrated that targeted appropriate behaviors can be increased through the direct manipulation of MOs.

\section{Stimulus Control}

Stimulus control is demonstrated when a particular behavior is reliably occasioned by specific antecedent stimuli (Sulzer-Azaroff \& Mayer, 
1991). In terms of the 3-term contingency, stimulus control describes a relationship between environment and behavior that consists of $\mathrm{A}$ (the antecedent) reliably occasioning $\mathrm{B}$ (the behavior), which results in $\mathrm{C}$ (the consequence or reinforcement). One way stimulus control can be established is by pairing specific responses with reinforcement only when they occur in the presence of specific antecedent stimuli, and withholding reinforcement when those specific responses are exhibited in the absence of the specific antecedent stimuli. This process is often programmed in laboratory research. However, it can also occur naturally as individuals are exposed to different stimulus contexts and their specific reinforcement schedules. For example, behavior may come under stimulus control due to the different ways a child's parents respond to his or her behavior.

In the presence of the father, problem behavior might always result in attention. Conversely, in the presence of the mother, problem behavior might have no differential consequence. If attention from care providers is a reinforcer, the child might begin engaging in problem behavior only in the presence of the father. In a similar fashion, punishment of a behavior in the presence of a specific stimulus might result in that behavior being inhibited in the presence of the stimulus. Using the same example, if the child's mother always delivered an aversive consequence (e.g., timeout or spanking) following problem behavior, but the father provided no differential consequence, the child might stop engaging in problem behavior in the presence of the mother only.

When stimulus control is apparent as exhibited by differential responding correlated with specific stimuli, treatment might focus on transferring stimulus control to improve behavior across stimulus contexts. Ray, Skinner, and Watson (1999) used stimulus control procedures to increase compliance exhibited by a five-year-old boy diagnosed with autism. During baseline, the investigators evaluated compliance when demands were delivered by the child's teacher as compared to when demands were delivered by the child's mother. The likelihood that the child would comply with demands was increased when his mother delivered demands as opposed to when his teacher delivered demands. This finding suggested that stimulus control had been established.

Using that information, the investigators next implemented a series of procedures in which the teacher was paired with the mother during demand situations. Initially, the child's mother delivered three demands and the teacher delivered one demand and compliance was observed at high rates with both adults. Over time, a fading procedure was used in which the teacher delivered an increasing number of the demands and the child's mother delivered fewer of the demands while compliance continued at high rates. Eventually, the child's mother was faded completely out of the demand situation, the teacher delivered all of the demands, and compliance continued at high rates. The results of Ray et al. (1999) suggested the fading procedure resulted in a transfer of stimulus control from the child's mother to the teacher.

In a similar study, Knoff (1984) used stimulus control procedures to treat problem behavior exhibited by two boys, 9 and 10 years of age, who engaged in aggression, disruption, and oppositional behavior. With each of the children, problem behavior was occurring at high rates in the presence 
of a paraprofessional during morning and noon recesses. Likewise, with each of the children, appropriate behavior was reliably occurring at high rates in the presence of their classroom teacher. The goal of the stimulus control procedures was to generalize the apparent stimulus control that the presence of the teacher was exerting over appropriate behavior to the paraprofessional during recess times. The procedure consisted of the teacher attending each recess period during the first week. High levels of appropriate behavior were immediately observed with each of the children during the first week. During subsequent weeks, the teacher spent fewer and fewer days attending recess periods until she was completely faded out. After the teacher was completely faded out of the recess, high levels of appropriate behavior continued to be observed with each of the children suggesting that stimulus control had been generalized or to the paraprofessional.

\section{Prompt Procedures}

Cooper et al. (2007) defined prompts as supplementary antecedent stimuli intended to occasion specific responses. Whereas response prompts (i.e., graduated guidance) target behavior, stimulus prompts target the antecedent conditions that exist prior to the occurrence of specific behavior (i.e., antecedents). Behavior analysts use stimulus prompts as auxiliaries to be removed over time as the intended behavior occurs more reliably in the presence of natural stimuli (discriminative stimuli). Prompts are often used during initial phases of treatment programs to facilitate the acquisition of specific responses. Following acquisition, the prompts can then be systematically faded so that naturally occurring stimuli will come to reliably occasion the acquired behavior.

Taylor and Levin (1998) and Shabani, Katz, Wilder, Beauchamp, Taylor, and Fischer (2002) each used a prompting procedure to promote social initiations with children with diagnoses of autism. The investigators used a tactile prompting device located in the children's pockets. Specifically, the device was programmed to vibrate for 3 to $5 \mathrm{~s}$ whenever the investigators activated it using a remote control. The investigators initially paired a vocal model with the tactile prompt to bring about social initiations, and then gradually faded the vocal model as the children independently exhibited social initiations following tactile prompts. The use of the vocal modeling and tactile prompts resulted in high rates of social initiations exhibited by the children across both studies.

In addition, Shabani et al. (2002) also attempted to fade the tactile prompt with two of the three participants by systematically reducing the frequency of the prompts over time. The results suggested that fading the tactile prompt was partially successful for each of the particiapants as social interactions continued, but at lower and more variable rates.

Rivera, Koorland, and Fueyo (2002) used picture prompts to promote sight word reading with a nine-year-old boy diagnosed with a learning disability. The picture prompts, which were generated by the child himself, were illustrated representatives of the targeted sight words. Initially, the experimenters reviewed with the child the meaning of each of the targeted sight words and had him generate illustrations for each of the words on 
large index cards. As the child's sight-word reading accuracy increased, the index cards on which he drew the illustrations were reduced and the colors that he was allowed to use were systematically lightened until the illustrations were successfully faded from the program. Even as the picture prompts were faded, the child continued to exhibit a high level of accurate sight-word reading.

\section{Choice}

Another antecedent-based intervention that has been demonstrated to be effective involves providing choice-making opportunities. Numerous studies have shown that providing choice can serve to decrease problem behavior and increase appropriate behavior including academic and vocational task engagement. Furthermore, choice has been conceptualized as a functional variable (i.e., a reinforcer for appropriate behavior) in and of itself rather than simply a means to identify highly preferred stimuli (Dunlap et al., 1994).

Dibley and Lim (1999) provided choice-making opportunities during treatment with a 15-year-old girl diagnosed with a severe intellectual disability. Choice-making opportunities were incorporated into various activities including meal-time routine, toileting routine, and leisure time activities for the purpose of increasing compliance and decreasing problem behaviors. During baseline, the adolescent was prompted to engage in each step that made up the respective activities and no choices were incorporated. During treatment, the adolescent was prompted to engage in each step that made up the respective activities with various opportunities for choice embedded throughout each of the activities. For example, during the toileting routine, the adolescent was provided with a choice between initiating the activity immediately or following a 10-min delay, basin or sink for hand washing, and hand-towel or hand dryer. When choices were provided, compliance was observed at higher levels and problem behavior was observed at lower levels when compared to baseline. These results were consistent across each of the three targeted activities.

Dunlap et al. (1994) incorporated choice-making opportunities into treatment programs for three young boys aged 11,11 , and 5 for the purpose of decreasing noncompliance and aggressive behavior. Two of the children received opportunities to make choices during instructional times in the form of menus containing several academic tasks. Choice-making opportunities for the third child were incorporated into reading time. Specifically, the child was allowed to pick a book from an array prior to storytime. When choices were provided, each child exhibited lower levels of noncompliance and problem behavior and task engagement was observed at higher levels than those observed during baseline.

\section{Combining Antecedent and Consequence-Based Treatments}

Often, more than one treatment is selected for implementation. Such treatment packages might be constructed of both antecedent and consequencebased components. Ringdahl et al. (2002) provided one such example. 
Treatment evaluated in that study combined DRA with instructional fading in the treatment escape-related problem behavior exhibited by a girl diagnosed with autism. The consequence portion of the treatment was a differential reinforcement schedule that specified the delivery of brief breaks contingent on appropriate compliance (i.e., completion without exhibiting problem behavior) with academic tasks. The antecedent component of the treatment was systematically increasing the number of instructions that were delivered during a 5-min work session, as long as problem behavior remained low, until a terminal goal of one instruction per min was achieved. Results of this study suggested that this combination of treatment components resulted in successful treatment with fewer occurrences of problem behavior than a consequence-based treatment (DRA) alone.

Marcus and Vollmer (1996) combined antecedent and consequencebased treatments in the treatment of SIB and aggression displayed by a young girl with developmental disabilities. Their investigation evaluated a treatment comprised of two components: NCR (antecedent) and differential reinforcement of compliance (consequence). Results of the study indicated that the treatment package was effective in reducing problem behavior. In addition, the treatment was effective in teaching the young girl how to use an alternative, appropriate communicative response to obtain the reinforcer that maintained problem behavior. The use of the package allowed for effective treatment while limiting some of the side effects (e.g., response bursts) sometimes observed during behavioral treatments with an extinction component.

\section{Generalization}

Generalization is one of the stated characteristics of applied behavior analysis (Baer et al., 1968). According to Cooper et al. (2007), generalization is a broad term that refers to a number of behavior change outcomes. During clinical application of ABA-based treatments, there is often an attempt to expand the effects of treatment from the clinical setting to the naturalistic environment (i.e., stimulus/setting generalization). Stimulus/setting generalization refers to the occurrence of a behavior under different conditions than which the behavior was acquired. Cooper et al. point out that this behavior change can occur without being directly taught. However, some behavior analysts attempt to facilitate this outcome through programming. Literature-based examples of generalization can be broken into two broad categories. Some studies describe the naturally occurring spread of effects across setting, time, and stimuli, whereas others describe systematic processes to achieve generalization.

Bonfiglio, Daly, Martens, Lin, and Corsaut (2004) described the effects of various reading interventions on the reading accuracy of a third-grade girl. The participant was exposed to performance-based, skills-based, and combined performance-based and skills-based reading interventions. Each treatment was demonstrated to improve reading behavior. The effects of treatment were noted across time and reading passages. These effects were achieved without specific programming. The authors hypothesized that generalization, particularly across passages, was a function (or, partially a function) of a fluency threshold. 
Eikeseth and Nesset (2003) described a treatment designed to improve vocal articulation exhibited by children with phonological disorder. As part of their treatment, the goal was to bring about mastery of a variety of target sounds (i.e., vocally produce the sounds without articulation errors). Toward this end, the two participants were exposed to a treatment that included sufficient response-exemplars. Specifically, a set of ten words was programmed to be used to teach the child the sound. During treatment, participants received tokens that could be exchanged for individually determined back-up reinforcers contingent on correct articulation or close approximations of the therapist's vocal model. Results suggested that both participants mastered each sound without needing to be exposed to all ten target words. The necessary number of words needed varied from one to eight. Thus, according to Eikeseth and Nesset, "after acquiring correct articulation of some words containing a particular target sound, other words containing the same target sound were subsequently echoed correctly without training” (p. 33-334). That is, generalized behavior change took place.

\section{EFFECTIVENESS RESEARCH}

Another s tated characteristic of applied behavior analysis is effectiveness (Baer et al., 1968). Although metaanalyses regarding the effectiveness of ABA-based treatments are difficult to identify, there are a number of studies that review the effectiveness of ABA-based strategies in the treatment of severe behavior problems exhibited by individuals with and without developmental disabilities. These reviews and summary papers can be placed into one of three broad categories: summaries of treatments for behavior associated with particular disorders (e.g., autism, ADHD), summaries of treatments for specific behavior problems (e.g., SIB, aberrant behavior, and stereotypy), and summaries of the effects of a specific treatment approach (e.g., NCR and FCT).

\section{Treatment of Behavior Challenges Associated with Particular Diagnoses}

Matson et al. (1996) provided a review of behavioral treatment strategies designed to address the challenging behavior exhibited by individuals with autism. Results of their review suggested that behavior analysts have used methods derived from the principles of operant conditioning to address a wide range of target behaviors exhibited by children diagnosed with autism including aberrant behavior, language, and social, daily living, and academic skills. These authors also found the percentage of interventions reported that used positive procedures outnumbered significantly the number of interventions that used aversive procedures. Olson and Houlihan (2000) reviewed behavioral treatments for challenging behaviors associated with Lesch-Nyhan disorder. The review suggested that in most cases, the use of behavioral treatments (i.e., DRO, DRI, extinction) were effective in treating self-injury exhibited by children with Lesch-Nyhan and that in many cases the results generalized to other settings and care providers. 


\section{Treatment of Specific Problem Behavior}

A number of other review papers have summarized ABA-based procedures as they pertain to the treatment of specific behavior problems. Iwata et al. (1994) reported the use function-based behavior analytic treatment to reduce self-injurious behavior (SIB) exhibited by adults and children with developmental disabilities. Effective treatment was defined as a treatment procedure that resulted in a decrease in problem behavior to below $10 \%$ of the baseline level for a given individual. Iwata et al. reported that, when interventions were based on identified functions of SIB, antecedent-based interventions were effective in $84.2 \%$ of reported cases, extinction was effective in $86.8 \%$ of reported cases, differential reinforcement was effective in $82.5 \%$ of reported cases, and punishment was effective in $88.2 \%$ of reported cases.

Similarly, Asmus et al. (2004) reported the treatment effects of function-based behavior analytic treatments in the reduction of aberrant behavior (SIB, aggression, stereotypy, destruction, and disruption) exhibited by adults and children with and without developmental disabilities. They reported an $80 \%$ decrease in aberrant behavior following the implementation of ABA-based treatments for $76 \%$ of the treated individuals. Rapp and Vollmer (2005) provided a summary of the literature concerning the treatment approaches to reducing stereotypy (i.e., repetitive behavior that serves no apparent social function). These authors concluded that there is ample support in the literature for the effectiveness of ABA-based treatments (both antecedent and consequence) in reducing stereotypy.

\section{Effectiveness of Specific Treatment Strategies}

Finally, several summaries have been published on the effectiveness of specific ABA-based approaches to treatment. Miltenberger, Fuqua, and Woods (1998) reported on the effectiveness of habit reversal methods for the treatment of target behaviors including tics, nervous habits, and stuttering. The authors suggested that habit reversal methods have been consistently demonstrated as effective even in the absence of identification of functions of the treated tics, habits, and stuttering. Carr et al. (2000) conducted a review of studies that evaluated the use of NCR in the treatment of aberrant behavior. The authors reported that NCR has been an effective treatment strategy for a variety of problem behaviors exhibited by individuals with developmental disabilities but included the caveat that more clinical research needs to be conducted in the area. Taken as a group, summaries provide a persuasive demonstration of the effectiveness of ABA-based treatments for a number of childhood behavior challenges exhibited by children with psychiatric and developmental disabilities.

\section{SUMMARY}

We have attempted to provide an overview of the conceptual basis for ABA-based treatments, a description of several of the more common of these treatments, and a brief discussion of their effectiveness. Applied behavior 
analysis-based treatment approaches have an established and effective history in the treatment of problem behavior and the establishment of appropriate behavior across a wide range of disabilities. The approach is not designed to treat the underlying disorder, per se. Instead, ABA-based treatments target specific behavioral symptoms indicative of an individual's diagnosis. Related strategies can be used to either establish new behavior or decrease existing problem behavior through an analytic process requiring an understanding of the antecedent and consequent variables affecting the target behavior. Although several approaches to behavior change exist, ABA-based treatments offer an evidence-based methodology with strong roots in basic and applied research. In our opinion, ABA represents a state-of-the-art approach to the development of behavior-change programs.

\section{REFERENCES}

Asmus, J. M., Ringdahl, J. E., Sellers, J. A., Call, N. A., Andelman, M. S., \& Wacker, D. P. (2004). Use of a short-term inpatient model to evaluate aberrant behavior: Outcome data summaries from 1996 to 2001. Journal of Applied Behavior Analysis, 37, 283-304.

Baer, D. M., Wolf, M. M., \& Risley, T. R. (1968). Some current dimensions of applied behavior analysis. Journal of Applied Behavior Analysis, 1, 91-97.

Berg, W. K. Peck, S., Wacker, D. P., Harding, J., McComas, J., Richman, D., \& Brown, K. (2000). The effects of presession exposure to attention on the results of assessments of attention as a reinforcer. Journal of Applied Behavior Analysis, 33, 463-477.

Bonfiglio, C. M., Daly, III, E. J., Martens, B. K., Rachel Lin, L. H., \& Corsaut, S. (2004). An experimental analysis of reading interventions: Generalization across instructional strategies, time, and passages. Journal of Applied Behavior Analysis, 37, 111-114.

Buzas, H. P., Ayllon, T., \& Collins, R. (1981). A behavioral approach to eliminate selfmutilative behavior in a Lesch-Nyhan patient. Journal of Mind and Behavior, 1, 47-56.

Carr, E. G., \& Durand, V. M. (1985). Reducing behavior problems through functional communication training. Journal of Applied Behavior Analysis, 18, 111-126.

Carr, J. E., Coriaty, S., Wilder, D. A., Gaunt, B. T., Dozier, C. L., Britton, L. N., Avina, C., \& Reed, C. L. (2000). A review of "noncontingent" reinforcement as treatment for the aberrant behavior of individuals with developmental disabilities. Research in Developmental Disabilities, 21, 377-391.

Conyers, C., Miltenberger, R., Maki, A., Barenz, R., Jurgens, M, Sailer, A., Haugen, M., \& Kopp, B. (2004). A comparison of response cost and differential reinforcement of other behavior to reduce disruptive behavior in a preschool classroom. Journal of Applied Behavior Analysis, 37, 411-415.

Cooper, J. O., Heron, T. E., \& Heward, W. L., (2007). Applied behavior analysis (3rd ed.). Upper Saddle River, NJ: Prentice-Hall.

Derby, K. M., Wacker, D. P., Berg, W., DeRaad, A., Ulrich, S., Asmus, J., Harding, J., Prouty, A., Laffey, P., \& Stoner, E. A. (1997). The long-term effects of functional communication training in home settings. Journal of Applied Behavior Analysis, 30, 507-531.

Dibley, S. \& Lim, L. (1999). Providing choice making opportunities within and between daily school routines. Journal of Behavioral Education, 9, 117-132.

Dietz, S. M., \& Repp, A. C. (1973). Decreasing classroom misbehavior through the use of DRL schedules of reinforcement. Journal of Applied Behavior Analysis, 6, 457-463.

Dunlap, G., dePerczel, M., Clarke, S., Wilson, D., Wright, S., White, R., \& Gomez, A. (1994). Choice making to promote adaptive behavior for students with emotional and behavioral challenges. Journal of Applied Behavior Analysis, 27, 505-518. 
Eikeseth, S. \& Nesset, R. (2003). Behavioral treatment of children with phonological disorder: The efficacy of vocal imitation and sufficient-response-exemplar training. Journal of Applied Behavior Analysis, 36, 325-337.

Falcomata, T. S., Roane, H. S., Hovanetz, A., N., Kettering, T. L., \& Keeney, K., M. (2004). An evaluation of response cost in the treatment of inappropriate vocalizations maintained by automatic reinforcement. Journal of Applied Behavior Analysis, $37,83-87$.

Field, C. E., Nash, H. M., Handwerk, M. L., \& Friman, P. C. (2004). A modification of the token economy for nonresponsive youth in family-style residential care. Behavior Modificaiton, 28, 438-457.

Foxx, R. M., \& Azrin, N. H. (1973). The elimination of autistic self-stimulatory behavior by overcorrection. Journal of Applied Behavior Analysis, 6, 1-14.

Friman, P. C. \& Altman, K. (1990). Parent use of DRI on high rate disruptive behavior: Direct and collateral benefits. Research in Developmental Disabilities, 2, 249-254.

Graff, R. B., Gibson, L., \& Galiatsatos, G. T., (2006). The impact of high- and low-preference stimuli on vocational and academic performances of youths with severe disabilities. Journal of Applied Behavior Analysis, 39, 131-135.

Hagopian, L. P., Contrucci Kuhn, S. A., Long, E. S., \& Rush, K. S. (2005). Schedule thinning following communication training: Using competing stimuli to enhance tolerance to decrements in reinforcer density. Journal of Applied Behavior Analysis, 38, 177-193.

Hagopian, L. P., Fisher, W. W., Sullivan, M. T., Acquisto, J., \& LeBlanc, L. A. (1998). Effectiveness of functional communication training with and without extinction and punishment: A summary of 21 inpatient cases. Journal of Applied Behavior Analysis, 31, 211-235.

Heward, W. L. (2005). Reasons applied behavior analysis is good for education and why those reasons have been insufficient. In W. L. Heward, T. E. Heron, N. A. Neef, S. M. Peterson, D. M. Sainato, G. Cartledge, R. Gardner, III, L. D. Peterson, S. B. Hersh, \& J. C. Dardig (Eds.), Focus on behavior analysis in education: Achievements, challenges, and opportunities (pp.316-348). Upper Saddle River, NJ: Merrill/Prentice Hall.

Iwata, B. A., Pace, G. M., Dorsey, M. F., Zarcone, J. R., Vollmer, T. R., Smith, R. G., Rodgers, T. A., Lerman, D. C., Shore, B. A., Mazaleski, J. L., Goh, H. L., Cowdery, G. E., Kalsher, M. J., McCosh, K. C., \& Willis, K. D. (1994)The functions of selfinjurious behavior: An experimental-epidemiological analysis. Journal of Applied Behavior Analysis, 27, 215-240.

Iwata, B. A., Pace, G. M., Kalsher, M. J., Cowdery, G. E., \& Cataldo, M. F. (1990). Experimental analysis and extinction of self-injurious escape behavior. Journal of Applied Behavior Analysis, 23, 11-27.

Kahng, S. W., Abt, K. A., \& Wilder, D. A. (2001). Treatment of self-injury correlated with mechanical restraints. Behavioral Interventions, 15, 105-110.

Kelley, M. E., Piazza, C. C., Fisher, W. W., \& Oberdorff, A. J. (2003). Acquisition of cup drinking using previously refused foods as positive and negative reinforcement. Journal of Applied Behavior Analysis, 36, 89-93.

Knoff, H. M. (1984). Stimulus control, paraprofessionals, and appropriate playground behavior. School Psychology Review, 13, 249-253.

Kodak, T., Grow, L., \& Northup, J. (2004). Functional analysis and treatment of elopement for a child with attention deficit hyperactivity disorder. Journal of Applied Behavior Analysis, 37, 229-232.

Lalli, J. S., Casey, S. D., \& Kates, K. (1997). Noncontingent reinforcement as treatment for severe problem behavior: Some procedural variations. Journal of Applied Behavior Analysis, 30, 127-137.

Lalli, J. S., Vollmer, T. R., Progar, P. R., Wright, C., Borrero, J., Daniel, D., Barthold, C. H., Tocco, K., \& May, W. (1999) Competition between positive and negative reinforcement in the treatment of escape behavior. Journal of Applied Behavior Analysis, 32, 285-296.

Laraway, S., Snycerski, S., Michael, J., \& Poling, A. (2003). Motivating operations and terms to describe them: Some further refinements. Journal of Applied Behavior Analysis, 36, 407-414. 
Lerman, D.C., Iwata, B.A., \& Wallace, M.D. (1999). Side effects of extinction: Prevalence of bursting and aggression during the treatment of self-injurious behavior. Journal of Applied Behavior Analysis, 32, 1-8.

Linscheid, T. R., Iwata, B. A., Ricketts, R. W., Williams, D. E., \& Griffin, J. C. (1990). Clinical evaluation of the self-injurious behavior inhibiting system (SIBIS). Journal of Applied Behavior Analysis, 23, 53-78.

Long, T. S., Miltenberger, R. G., \& Rapp, J. T. (1999) Simplified habit reversal plus adjunct contingencies in the treatment of thumb sucking and hair pulling in a young child. Child \& Family Behavior Therapy, 21, 45-58.

Luiselli, J. K. (1991) Acquisition of self-feeding in a child with Lowe's syndrome. Journal of Developmental and Physical Disabilities, 3, 181-189.

Magee, S. K., \& Ellis, J. (2000). Extinction effects during the assessment of multiple problem behaviors. Journal of Applied Behavior Analysis, 33, 313-316.

Mangus, B., Henderson, H., \& French, R. (1986). Implementation of a token economy by peer tutors to increase on-task physical activity time of autistic children. Perceptual and Motor Skills, 1, 97-98.

Marcus, B. A., \& Vollmer, T. R. (1996). Combining noncontingent reinforcement and differential reinforcement schedules as treatment for aberrant behavior. Journal of Applied Behavior Analysis, 29, 43-51.

Matson, J. L., Benavidez, D. A., Stabinsky Compton, L., Paclawskyi, T., \& Baglio, C. (1996). Behavioral treatment of autistic persons: A review of research from 1980 to the present. Research in Developmental Disabilities, 17, 433-465.

Michael, J. (1982). Distinguishing between discriminative and motivational functions of stimuli. Journal of the Experimental Analysis of Behavior, 1, 149-155.

Miltenberger, R. G., Fuqua, R. W., \& Woods, D. W. (1998). Applying behavior analysis to clinical problems: Review and analysis of habit reversal. Journal of Applied Behavior Analysis, 31, 447-469.

Olson, L. \& Houlihan, D. (2000). A review of behavioral treatments used for LeschNyhan Syndrome. Behavior Modification, 24, 202-222.

Rapp, J. T., \& Vollmer, T. R. (2005). Stereotypy I: A review of behavioral assessment and treatment. Research in Developmental Disabilities, 26, 527-547.

Ray, K. P., Skinner, C. H., \& Watson, T. S. (1999). Transferring stimulus control via momentum to increase compliance in a student with autism: A demonstration of collaborative consultation. School Psychology Review, 28, 622-628.

Reed, G. K., Ringdahl, J. E., Wacker, D. P., Barretto, A., \& Andelman, M. S. (2005). The effects of fixed-time and contingent schedules of negative reinforcement on compliance and aberrant behavior. Research in Developmental Disabilities, 3, 281295.

Ringdahl, J. E., Andelman, M. S., Kitsukawa, K., Winborn, L. C., Barretto, A., \& Wacker, D. P. (2002). Evaluation and treatment of covert stereotypy. Behavioral Interventions, $17,43-49$.

Ringdahl, J. E. Kitsukawa, K. Andelman, M. S. Call, N. Winborn, L. Barretto, A., \& Reed, G. K. (2002). Differential reinforcement with and without instructional fading. Journal of Applied Behavior Analysis, 35, 291-294.

Ringdahl, J. E., Vollmer, T. R., Borrero, J. C., \& Connell, J. E. (2001). Fixed-time schedule effects as a function of baseline reinforcement rate. Journal of Applied Behavior Analysis, 34, 1-15.

Rivera, M. O., Koorland, M. A., \& Fueyo, V. (2002). Teaching sight words to a student with learning disabilities. Education and Treatment of Children, 25, 197-207.

Rolider, A., \& Van Houten, R. (1985). Treatment of constipation-caused encopresis by a negative reinforcement procedure. Journal of Behavior Therapy and Experimental Psychiatry, 16, 67-70.

Rush, K. S., Crocket, J. L., \& Hagopian, L. P. (2001). An analysis of the selective effects of NCR with punishment targeting problem behavior associated with positive affect. Behavioral Interventions, 16, 127-135.

Shabani, D. B., Katz, R. C., Wilder, D. A., Beauchamp, K., Taylor, C. R., \& Fischer, K. J. (2002). Increasing social initiations in children with autism: Effects of a tactile prompt. Journal of Applied Behavior Analysis, 35, 79-83. 
Singh, N. N., Watson, J. E., \& Winton, A. S. W. (1986). Treating self-injury: Water mist spray versus facial screening or forced arm exercise. Journal of Applied Behavior Analysis, 19, 403-410.

Sulzer-Azaroff, B., \& Mayer, G. R. (1991). Behavior analysis for lasting change. New York: Harcourt Brace College.

Taylor, B. A., Hoch, H., Potter, B., Rodriguez, A., Spinnato, D., \& Kalaigan, M. (2005). Manipulating establishing operations to promote initiations toward peers in children with autism. Research in Developmental Disabilities, 26, 385-392.

Taylor, B. A., \& Levin, L. (1998). Teaching a student with autism to make verbal initiations: Effects of a tactile prompt. Journal of Applied Behavior Analysis, 31, 651654.

Vollmer, T. R. (2002). Punishment happens: Some comments on Lerman and Vorndran's review. Journal of Applied Behavior Analysis, 35, 469-473.

Watson, T. S., \& Sterling, H. E. (1998). Brief functional analysis and treatment of a vocal tic. Journal of Applied Behavior Analysis, 31, 471-474.

Wright, C. S., \& Vollmer, T. R. (2002). Evaluation of a treatment package to reduce rapid eating. Journal of Applied Behavior Analysis, 35, 89-93. 